\title{
THE SMALL QUANTUM COHOMOLOGY OF THE CAYLEY GRASSMANNIAN
}

\author{
VLADIMIRO BENEDETTI AND LAURENT MANIVEL
}

\begin{abstract}
We compute the small cohomology ring of the Cayley Grassmannian, that parametrizes four-dimensional subalgebras of the complexified octonions. We show that all the Gromov-Witten invariants in the multiplication table of the Schubert classes are non negative and deduce Golyshev's conjecture $\mathcal{O}$ holds true for this variety. We also check that the quantum cohomology is semisimple and that there exists, as predicted by Dubrovin's conjecture, an exceptional collection of maximal length in the derived category.
\end{abstract}

\section{The Setup}

The Cayley Grassmannian $C G$ is a closed subvariety of the complex Grassmannian $G(3,7) \simeq G(4,7)$, which can be described as follows:

(1) either as the subvariety of $G(3,7)$ parametrizing (the imaginary parts of) the four-dimensional subalgebras of the complexified octonions,

(2) or as the zero-locus of a general section of the vector bundle $\wedge^{3} T^{*}$, where $T$ denotes the tautological bundle on $G(4,7)$.

The equivalence of these two descriptions comes from the facts that a global section of $\wedge^{3} T^{*}$ is a skew-symmetric three-form in seven variables, and that the stabilizer of a general such form is a copy of $G_{2}$, the automorphism group of the octonions.

The reader will find in $\mathrm{Ma}$ more details on the geometry of the Cayley Grassmannian; let us just recall that it is a Fano eightfold of Picard number one and index four. Its equivariant cohomology ring (with respect to a maximal torus in $G_{2}$ ) has been computed in [Ma, 4.2] with the help of the classical localization techniques. These computations imply that the ordinary cohomology ring is generated (over the rational numbers) by the hyperplane class $\sigma_{1}$ and a codimension two class $\sigma_{2}$. The even Betti numbers are 1,1,2, 2, 3, 2, 2,1,1 and there is an integral basis of Schubert classes for which we keep the notations of $\mathrm{Ma}$. The so called Chevalley formula for the product by the hyperplane class is encoded in the graph below, where the number of edges between two classes is the coefficient of the larger degree class in the hyperplane product of the other one. Moreover the rational cohomology ring is defined by two relations in degree five and six:

Proposition 1.1. The rational cohomology ring of $C G$ is

$$
H^{*}(C G, \mathbb{Q})=\mathbb{Q}\left[\sigma_{1}, \sigma_{2}\right] /\left\langle\sigma_{1}^{5}-5 \sigma_{1}^{3} \sigma_{2}+6 \sigma_{1} \sigma_{2}^{2}, 16 \sigma_{2}^{3}-27 \sigma_{1}^{2} \sigma_{2}^{2}+9 \sigma_{1}^{4} \sigma_{2}\right\rangle .
$$

We will denote these two relations by $R_{5}$ and $R_{6}$. The full multiplication table is given in [Ma, 4.3].

Beware! There is a typo in this table, a coefficient 3 in $\sigma_{5}^{\prime} \sigma_{2}=3 \sigma_{7}$ is missing. 


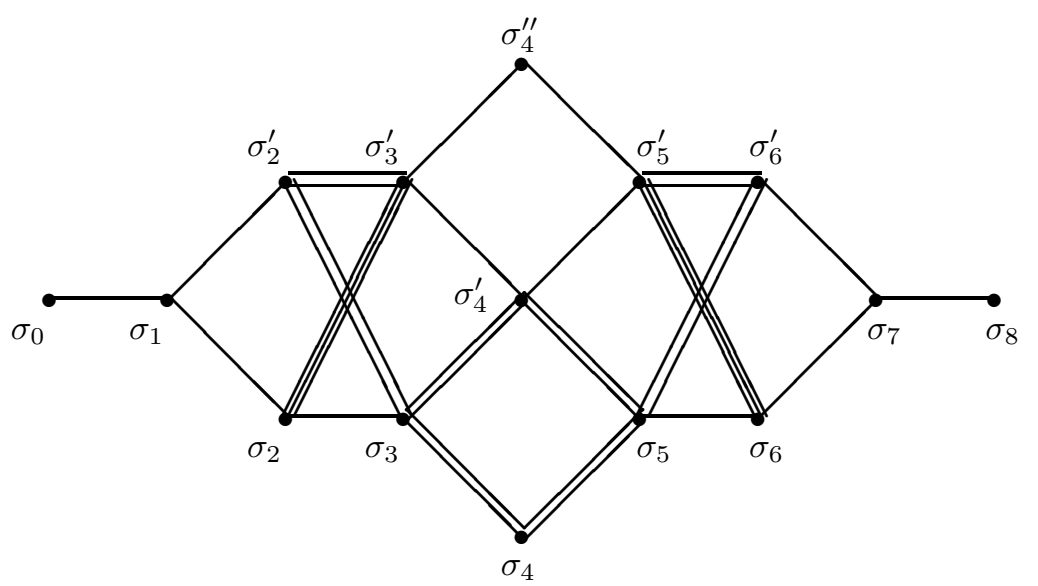

The Bruhat graph of $C G$

\section{How to COMPUte The QUANTUm COHOMOlOGY RING}

2.1. Deforming the cohomology ring. The small quantum cohomology ring of the Cayley Grassmannian, which we intend to determine, is a deformation of its ordinary cohomology ring. The quantum parameter $q$ has degree four, the Fano index of $C G$. The quantum products of two Schubert classes are of the form

$$
\sigma \sigma^{\prime}=\sigma \cup \sigma^{\prime}+\sum_{d>0} q^{d} \sum_{\tau} I_{d}\left(\sigma, \sigma^{\prime}, \tau\right) \tau^{\vee}
$$

where the sum is over the Schubert classes $\tau$ whose Poincaré dual class has degree $\operatorname{deg}\left(\tau^{\vee}\right)=\operatorname{deg}(\sigma)+\operatorname{deg}\left(\sigma^{\prime}\right)-4 d$ (recall from Ma that the basis of Schubert classes is self-dual, up to order). Moreover $I_{d}\left(\sigma, \sigma^{\prime}, \tau\right)$ is the three-points degree $d$ GromovWitten invariant associated to the three Schubert classes $\sigma, \sigma^{\prime}, \tau$. Note that, since $C G$ has dimension eight, $d$ does never exceed four.

A first useful observation is that, by the results of [TS], the small quantum cohomology ring has a presentation of the form

$$
Q H^{*}(C G, \mathbb{Q})=\mathbb{Q}\left[\sigma_{1}, \sigma_{2}, q\right] /\left\langle R_{5}(q), R_{6}(q)\right\rangle,
$$

where the relations $R_{5}(q)$ and $R_{6}(q)$ are deformations of $R_{5}$ and $R_{6}$ : in fact we can just consider the latter relations, and evaluate them in the quantum cohomology ring rather than in ordinary cohomology. For degree reasons we will obtain deformed relations of the form

$$
R_{5}(q)=R_{5}+q Q_{1}, \quad R_{6}(q)=R_{6}+q Q_{2} .
$$

Since there is no term with degree in $q$ bigger than one, these relations are completely determined by the Gromov-Witten invariants of degree one. In fact, as we are going to see, this turns out to be true for the full quantum product.

2.2. Degree one Gromov-Witten invariants are enough. In order to determine the full quantum products, the natural strategy would be to:

(1) find the quantum relations $R_{5}(q), R_{6}(q)$;

(2) express the Schubert classes as polynomials in $\sigma_{1}, \sigma_{2}$ and $q$ : these are the quantum Giambelli formulas; 
(3) compute the products of the Schubert classes by $\sigma_{1}$ and $\sigma_{2}$ : these are the quantum Pieri classes.

The quantum products by $\sigma_{1}$ are given by the quantum Chevalley formulas. Because of the symmetries of the Gromov-Witten invariants, these products must be of the following form:

$$
\begin{aligned}
& \sigma_{1} \sigma_{1}=\sigma_{2}+\sigma_{2}^{\prime} \\
& \sigma_{2} \sigma_{1}=\sigma_{3}+3 \sigma_{3}^{\prime} \\
& \sigma_{2}^{\prime} \sigma_{1}=2 \sigma_{3}+2 \sigma_{3}^{\prime} \\
& \sigma_{3} \sigma_{1}=2 \sigma_{4}+2 \sigma_{4}^{\prime}+a_{3} q \\
& \sigma_{3}^{\prime} \sigma_{1}=\sigma_{4}^{\prime}+\sigma_{4}^{\prime \prime}+a_{3}^{\prime} q \\
& \sigma_{4} \sigma_{1}=2 \sigma_{5}+a_{4} q \sigma_{1} \\
& \sigma_{4}^{\prime} \sigma_{1}=2 \sigma_{5}+\sigma_{5}^{\prime}+a_{4}^{\prime} q \sigma_{1} \\
& \sigma_{4}^{\prime \prime} \sigma_{1}=\sigma_{5}^{\prime}+a_{4}^{\prime \prime} q \sigma_{1} \\
& \sigma_{5} \sigma_{1}=\sigma_{6}+2 \sigma_{6}^{\prime}+a_{5} q \sigma_{2}+b_{5} q \sigma_{2}^{\prime} \\
& \sigma_{5}^{\prime} \sigma_{1}=3 \sigma_{6}+2 \sigma_{6}^{\prime}+a_{5}^{\prime} q \sigma_{2}+b_{5}^{\prime} q \sigma_{2}^{\prime} \\
& \sigma_{6} \sigma_{1}=\sigma_{7}+a_{5} q \sigma_{3}+a_{5}^{\prime} q \sigma_{3}^{\prime} \\
& \sigma_{6}^{\prime} \sigma_{1}=\sigma_{7}+b_{5} q \sigma_{3}+b_{5}^{\prime} q \sigma_{3}^{\prime} \\
& \sigma_{7} \sigma_{1}=\sigma_{8}+a_{4} q \sigma_{4}+a_{4}^{\prime} q \sigma_{4}^{\prime}+a_{4}^{\prime \prime} q \sigma_{4}^{\prime \prime}+a_{7} q^{2} \\
& \sigma_{8} \sigma_{1}=a_{3} q \sigma_{5}+a_{3}^{\prime} q \sigma_{5}^{\prime}+a_{7} q^{2} \sigma_{1} .
\end{aligned}
$$

There are ten unknowns to compute; all of them are degree one Gromov-Witten invariants, except $a_{7}$. Suppose we have computed them; then we can almost deduce the quantum Giambelli formulas, because most Schubert classes belong to the image of the multiplication map by $\sigma_{1}$. To be precise, in ordinary cohomology the image of this multiplication map has codimension two; in order to generate the full cohomology ring, we just need to add for example $\sigma_{2}$ and $\sigma_{4}$, or $\sigma_{2}$ and $\sigma_{2}^{2}$. In particular, from quantum Chevalley and the quantum $\sigma_{2}^{2}$, we will be able to deduce quantum Giambelli. Note that the computation of $\sigma_{2}^{2}$ only involves a degree one Gromov-Witten invariant.

Once we have quantum Giambelli, we can compute inductively the products by $\sigma_{2}$, just using the fact that if a class $\sigma$ satisfies $\sigma=\tau \sigma_{1}$ for some other class $\tau$, then $\sigma \sigma_{2}$ can obviously be deduced from $\tau \sigma_{2}$ and quantum Chevalley. The missing ingredient is a formula for $\sigma_{4} \sigma_{2}$, or equivalently $\sigma_{2}^{3}$. Again the computation of the quantum product $\sigma_{4} \sigma_{2}$ only involves degree one Gromov-Witten invariants.

Finally, the only invariant we used previously which is not of degree one is $a_{7}$. From the quantum Giambelli formula in degree at most seven and the expression of $\sigma_{7} \sigma_{1}$ above, we can at this point express $\sigma_{8}$ in terms of $\sigma_{1}, \sigma_{2}, q, a_{7}$. But then we can deduce $\sigma_{8} \sigma_{1}$ as a linear combination of Schubert classes, and comparing with the formula above, this yields a non trivial equation in $a_{7}$. So $a_{7}$ is also determined by the other coefficients. (In fact we will check that $a_{7}=0$.) We have proved:

Lemma 2.1. The quantum cohomology ring $Q H^{*}(C G, \mathbb{Q})$ is determined by:

(1) the quantum Chevalley formula, up to degree seven;

(2) the quantum products $\sigma_{2}^{2}$ and $\sigma_{4} \sigma_{2}$.

In particular it is completely determined by degree one Gromov-Witten invariants.

2.3. Enumerativity. Homogeneous varieties have the nice property that their Gromov-Witten invariants are enumerative: they can be effectively computed as numbers of rational curves touching suitable collections of Schubert varieties in general position. This is definitely no longer the case for non homogeneous varieties, 
where certain Gromov-Witten invariants can be negative (and even certain classical intersection numbers). Fortunately, enumerativity can be preserved in certain specific situations. We will mainly use the following result from GPPS] (Theorem $3.3)$ :

Proposition 2.2. Let $X$ be a smooth projective variety, with an action of a reductive group $G$ that has only finitely many orbits. Let $\gamma_{1}, \ldots, \gamma_{k}$ be cohomology classes of degree bigger than one, represented by subvarieties $Y_{1}, \ldots, Y_{k}$ of $X$ that are transverse to the orbits.

Suppose moreover that the moduli space $M_{d, k}(X)$ of stable maps of genus zero and degree $d$ with $k$ marked points is irreducible, and such that the general such curve intersects the open orbit of $X$.

Then the Gromov-Witten invariant $I_{d}\left(\gamma_{1}, \ldots, \gamma_{k}\right)$ can be computed as the number of stable curves of degree $d$ that intersect general $G$-translates of $Y_{1}, \ldots, Y_{k}$.

In the sequel we will only apply this statement to $d=1$ and $k=2$ or $k=3$. Of course the Schubert varieties themselves are in general not transverse to the orbits of $C G$, so we need to be careful. We will show in the next section that general lines and general planes through a general point of $C G$ satisfy this hypothesis.

Alternatively, we can use Schubert varieties on the ambient Grassmannian $G(4,7)$, since the homogeneity of the latter allows to put their general $P G L_{7}$-translates in general position with any finite collection of subvarieties, in particular with the orbits in $C G$ (and $C G$ itself). This means that the intersections with $C G$ of general Schubert varieties in $G(4,7)$ will have the required properties.

The cohomology classes of these intersections are given by the restrictions of Schubert classes, which were computed in $[\mathrm{Ma}$, Proposition 4.7]. For future use we recall the results. Let us fix a complete flag $0=V_{0} \subset V_{1} \subset \cdots \subset V_{7} \cong \mathbb{C}^{7}$. For $\lambda=\left(\lambda_{1} \geq \cdots \geq \lambda_{4}\right)$ an integer sequence with $\lambda_{1} \leq 3$ and $\lambda_{4} \geq 0$, we have the usual Schubert variety

$$
X_{\lambda}=\left\{W \in G(4,7) \text { s.t. } \operatorname{dim}\left(W \cap V_{3-\lambda_{j}+j}\right) \geq j \text { for } 1 \leq j \leq 4\right\},
$$

of codimension $\sum_{i} \lambda_{i}$ inside $G(4,7)$. We denote its cohomology class by $\tau_{\lambda}$. In particular $\tau_{1}$ is the hyperplane class, whose restriction to $C G$ is just $\sigma_{1}$. The remaining pull-backs, up to degree six, are as follows.

Proposition 2.3. Let $\iota: C G \hookrightarrow G(4,7)$ be the natural embedding. Then:

$$
\begin{gathered}
\iota^{*} \tau_{2}=\sigma_{2}, \quad \iota^{*} \tau_{11}=\sigma_{2}^{\prime}, \\
\iota^{*} \tau_{3}=\sigma_{3}^{\prime}, \quad \iota^{*} \tau_{111}=\sigma_{3}, \\
\iota^{*} \tau_{1111}=\sigma_{4}, \quad \iota^{*} \tau_{211}=\sigma_{4}+2 \sigma_{4}^{\prime}, \\
\iota^{*} \tau_{22}=\sigma_{4}+\sigma_{4}^{\prime}+\sigma_{4}^{\prime \prime}, \quad \iota^{*} \tau_{31}=\sigma_{4}^{\prime}+\sigma_{4}^{\prime \prime}, \\
\iota^{*} \tau_{2111}=2 \sigma_{5}, \quad \iota^{*} \tau_{221}=3 \sigma_{5}+\sigma_{5}^{\prime}, \quad \iota^{*} \tau_{311}=\iota^{*} \tau_{32}=\sigma_{5}+\sigma_{5}^{\prime}, \\
\iota^{*} \tau_{2211}=\sigma_{6}+3 \sigma_{6}^{\prime}, \quad \iota^{*} \tau_{222}=2 \sigma_{6}+2 \sigma_{6}^{\prime}, \\
\iota^{*} \tau_{321}=3 \sigma_{6}+3 \sigma_{6}^{\prime}, \quad \iota^{*} \tau_{33}=\iota^{*} \tau_{3111}=\sigma_{6}+\sigma_{6}^{\prime} .
\end{gathered}
$$

Beware! In [Ma, Proposition 4.7], $\iota^{*} \tau_{2}$ and $\iota^{*} \tau_{11}$ have been interchanged.

\section{A Bit of GEOMETRY}

In this section we study lines and planes on $C G$. We denote by $\Omega$ a general three-form on the seven dimensional vector space $V_{7}$. One can then find a basis of 
$V_{7}$ (a basis of eigenvectors for a maximal torus of the copy if $G_{2}$ that stabilizes $\Omega$ ) for which $\Omega$ can be written as

$\Omega=v_{0} \wedge v_{\alpha} \wedge v_{-\alpha}+v_{0} \wedge v_{\beta} \wedge v_{-\beta}+v_{0} \wedge v_{\gamma} \wedge v_{-\gamma}+v_{\alpha} \wedge v_{\beta} \wedge v_{\gamma}+v_{-\alpha} \wedge v_{-\beta} \wedge v_{-\gamma}$.

Moreover there is a $G_{2}$-invariant quadratic form on $V_{7}$, that one can write as

$$
q=v_{0}^{2}+v_{\alpha} v_{-\alpha}+v_{\beta} v_{-\beta}+v_{\gamma} v_{-\gamma} .
$$

See [Ma, section 2.1] for more details.

3.1. Lines in the Cayley Grassmannian. Recall that the variety $F_{1}(G)$ of lines in the Grassmannian $G$ is the flag variety $F(3,5,7)$ parametrizing flags of subspaces $V_{3} \subset V_{5} \subset V_{7}$. Its two projections $p_{3}$ and $p_{5}$ onto $G(3,7)$ and $G(5,7)$ are locally trivial, with Grassmannians $G(2,4)$ and $G(3,5)$ as respective fibers.

Proposition 3.1. The variety $F_{1}(C G)$ of lines in $C G$ is a smooth subvariety of $F_{1}(G)$, of the expected dimension 9 .

Proof. Let us denote by $T_{3}$ and $T_{5}$ the tautological bundles of rank 3 and 5 on $F_{1}(G)$. By restriction, the vector bundle $\wedge^{2} T_{3}^{*} \otimes T_{5}^{*}$ maps to $\wedge^{2} T_{3}^{*} \otimes T_{3}^{*}$. Let $E$ denote the pre-image of $\wedge^{3} T_{3}^{*} \subset \wedge^{2} T_{3}^{*} \otimes T_{3}^{*}$. There is an exact sequence

$$
0 \rightarrow \wedge^{2} T_{3}^{*} \otimes\left(T_{5} / T_{3}\right)^{*} \rightarrow E \rightarrow \wedge^{3} T_{3}^{*} \rightarrow 0 .
$$

By the Borel-Weil theorem $H^{0}\left(F_{1}(G), \wedge^{2} T_{3}^{*} \otimes\left(T_{5} / T_{3}\right)^{*}\right)=0$ and $H^{0}\left(F_{1}(G), \wedge^{3} T_{3}^{*}\right)=$ $\wedge^{3} V_{7}^{*}$. Therefore $H^{0}\left(F_{1}(G), E\right)=\wedge^{3} V_{7}^{*}$. Our three-form $\Omega$ thus defines a general section of $E$, whose zero-locus is exactly $F_{1}(C G)$. The statement follows from generic smoothness since $E$ is globally generated. Indeed, let us choose a basis $e_{1}, \ldots, e_{7}$ of $V_{7}$ such that $e_{1}, \ldots, e_{3}$ is a basis of $T_{3}$ and $e_{1}, \ldots, e_{5}$ a basis of $T_{5}$. By choosing arbitrarily the coefficients $\Psi_{123}$ and $\Psi_{i j k}$ for $i, j \leq 3<k \leq 5$ of a three-form $\Psi$, which we can do freely, we generate the whole fiber of $E$.

Now let us consider the set of lines in the Cayley Grassmannian passing through a given point $p \in C G$, corresponding to $A_{4} \subset V_{7}$. Such a line is given by a pair $\left(V_{3}, V_{5}\right)$ such that $V_{3} \subset A_{4} \subset V_{5}$ and $\Omega\left(V_{3}, V_{3}, V_{5} / A_{4}\right)=0$ (note that $\Omega\left(V_{3}, V_{3}, A_{4}\right)=0$ since $\Omega$ vanishes on $A_{4}$ ). Focusing on $V_{5}$, consider the map

$$
\theta: V_{5} / A_{4} \longrightarrow \wedge^{2} A_{4}^{*}
$$

induced by $\Omega$. Note that $\theta$ cannot be zero, since otherwise $\Omega$ would vanish on $V_{5}$. This condition would define a codimension ten subvariety of $G\left(5, V_{7}\right)$, stable under $G_{2}$, hence a collection of fixed points: but there is none. So for a suitable $V_{3}$ to exist, we need the image of $\theta$ to be generated by a rank two form; then $V_{3}$ must contain the kernel of this rank two form, hence varies in a $\mathbb{P}^{1}$.

One can compute explicitly $\theta$ at three points $p$ representing the three orbits in $C G$. The conclusion is that the locus in $\mathbb{P}\left(V_{7} / A_{4}\right)$ defined by the condition that $\theta$ drops rank is either:

(1) a smooth conic if $p$ belongs to the open orbit,

(2) a reducible conic if $p$ belongs to the codimension one orbit,

(3) the whole plane if $p$ belongs to the closed orbit.

From this we deduce: 


\section{Proposition 3.2.}

(1) The variety of lines passing through a general point $p=\left[A_{4}\right]$ of $C G$ is a copy of $\mathbb{P}^{1} \times \mathbb{P}^{1}$, embedded inside $\mathbb{P}\left(A_{4}^{*}\right) \times \mathbb{P}\left(V_{7} / A_{4}\right)$ by a linear system of type $|\mathcal{O}(1,1)| \otimes|\mathcal{O}(2,0)|$.

(2) The variety $F_{1}(C G)$ is irreducible.

Proof. The first assertion is the result of a direct computation. For the second assertion, consider the point-line incidence variety $I \subset C G \times F_{1}(C G)$. On the one hand, its projection to $F_{1}(C G)$ is a $\mathbb{P}^{1}$-bundle, so $I$ is smooth of dimension 10 , and irreducible if and only if $F_{1}(C G)$ is irreducible. On the other hand, the fibers of its projection to $C G$ are smooth quadratic surfaces over the open orbit, and surfaces or threefolds over the other points. This implies that the preimage in $I$ of the open orbit is irreducible, and what remains is too small to generate another dimension ten component.

Remark. Recall from $\mathrm{Ma}$ that the stabilizer of a general point in $C G$ is isomorphic to $S L_{2} \times S L_{2}$. This stabilizer acts transitively on the quadratic surface that parametrizes the lines through this point. As a consequence, $G_{2}$ has an open orbit in $F_{1}(C G)$. More precisely, $G_{2}$ acts transitively on the space of lines meeting the open orbit of $C G$.

3.2. Planes in the Cayley Grassmannian. In order to simplify the computations of certain Gromov-Witten invariants, it will be useful to understand the planes in the Cayley Grassmannian. Indeed the two degree six Schubert classes $\sigma_{6}$ and $\sigma_{6}^{\prime}$ both represent planes contained in $C G$.

The Grassmannian $G=G(4,7)$ containes two different kind of planes, $\alpha$-planes parametrized by $F(3,6,7)$ and $\beta$-planes parametrized by $F(2,5,7)$. A $\beta$-plane is made of spaces contained in a codimension two subspace of $V_{7}$, so it cannot meet a class $\iota^{*} \tau_{2}=\sigma_{2}$. Since according to $\mathrm{Ma} \sigma_{6} \sigma_{2} \neq 0$ but $\sigma_{6}^{\prime} \sigma_{2}=0$, we deduce:

Lemma 3.3. The class of an $\alpha$-plane in $C G$ is $\sigma_{6}$, the class of a $\beta$-plane is $\sigma_{6}^{\prime}$.

Let us discuss these planes separately.

Proposition 3.4. The family of $\alpha$-planes in $C G$ is parametrized by the quadric $\mathbb{Q}^{5}$. There is no $\alpha$-plane through the general point of $C G$.

Proof. An $\alpha$-plane is defined by a pair $\left(V_{3} \subset V_{6}\right)$ of subspaces of $V_{7}$. It is contained in $C G$ if and only if $\Omega\left(V_{3}, V_{3}, V_{3}\right)=0$ and $\Omega\left(V_{3}, V_{3}, V_{6}\right)=0$. If we denote by $\omega$ the restriction of $\Omega$ to $V_{6}$, the latter condition means that $\omega$ belongs to $\wedge^{2} V_{3}^{\perp} \wedge V_{6}^{*}$, which is the tangent space to the Grassmannian $G\left(3, V_{6}^{*}\right)$ at $V_{3}^{\perp}$. In particular $\omega$ is not a generic three-form.

There are only two $G_{2}$-orbits of hyperplanes in $V_{7}$ : the orthogonal line (with respect to the invariant quadratic form) can be isotropic or not. Let us choose representatives of these orbits.

A non isotropic vector is $e_{0}$, its orthogonal being $V_{6}=\left\langle e_{\alpha}, e_{\beta}, e_{\gamma}, e_{-\alpha}, e_{-\beta}, e_{-\gamma}\right\rangle$. The restriction of $\Omega$ to this hyperplane is $\omega=v_{\alpha} \wedge v_{\beta} \wedge v_{\gamma}+v_{-\alpha} \wedge v_{-\beta} \wedge v_{-\gamma}$, a generic three-form.

An isotropic vector is $e_{\alpha}$, its orthogonal being $V_{6}=\left\langle e_{0}, e_{\alpha}, e_{\beta}, e_{\gamma}, e_{-\beta}, e_{-\gamma}\right\rangle$. The restriction of $\Omega$ to this hyperplane is $\omega=v_{0} \wedge v_{\beta} \wedge v_{-\beta}+v_{0} \wedge v_{\gamma} \wedge v_{-\gamma}+v_{\alpha} \wedge v_{\beta} \wedge v_{\gamma}$, which belongs to $\wedge^{2} V_{3}^{\perp} \wedge V_{6}^{*}$ only for $V_{3}^{\perp}=\left\langle v_{0}, v_{\beta}, v_{\gamma}\right\rangle$. Note that $V_{3}$ contains $e_{\alpha}$, so that any point $A$ in the $\alpha$-plane defined by $V_{6}$ verifies $V_{6}^{\perp} \subset A \subset V_{6}$. 
As a consequence, the restriction of the invariant quadratic form on $A$ must be degenerate; equivalently, by [Ma, Proposition 3.1], $A$ does not belong to the open orbit in $C G$.

Proposition 3.5. The family of $\beta$-planes in $C G$ has dimension seven. There is a conic of $\beta$-planes passing through the general point of $C G$. Moreover, a generic $\beta$-plane is transverse to the orbit stratification.

Proof. A $\beta$-plane is defined by a pair $\left(V_{2} \subset V_{5}\right)$ of subspaces of $V_{7}$. It is contained in $C G$ if and only if $\Omega\left(V_{2}, V_{2}, V_{5}\right)=0$ and $\Omega\left(V_{2}, V_{5}, V_{5}\right)=0$.

Let us describe the $\beta$-planes passing through a general point $p$ of $C G$, which we choose to be the point defined by $A=\left\langle e_{\alpha}, e_{\beta}, e_{-\alpha}, e_{-\beta}\right\rangle$. Suppose that $V_{5}$ is generated by $A$ and $a=x e_{0}+y e_{\gamma}+z e_{-\gamma}$. The conditions on $V_{2} \subset A$ now restrict to $\Omega\left(V_{2}, A, a\right)=0$, which means that $V_{2}$ must be contained in the kernel of the four linear forms $\Omega\left(a, e_{\alpha}, \bullet\right)=x v_{-\alpha}+y v_{\beta}, \Omega\left(a, e_{\beta}, \bullet\right)=x v_{-\beta}-y v_{\alpha}, \Omega\left(a, e_{-\alpha}, \bullet\right)=$ $-x v_{\alpha}+z v_{-\beta}, \Omega\left(a, e_{-\beta}, \bullet\right)=-x v_{\beta}-z v_{-\alpha}$. This systems of linear forms has rank four in general, and rank two if $x^{2}=y z$. There is therefore a conic of $\beta$-planes through a general point of $C G$, from which one can deduce that there is a seven dimensional family of $\beta$-planes on $C G$.

Let us choose for example $V_{5}=\left\langle e_{\alpha}, e_{\beta}, e_{-\alpha}, e_{-\beta}, e_{\gamma}\right\rangle$. Then $V_{2}=\left\langle e_{-\alpha}, e_{-\beta}\right\rangle$ and our plane $V_{2} \subset A \subset V_{5}$ can be described by $A^{\perp}=\left\langle e_{0}, e_{\gamma}, u e_{-\alpha}+v e_{-\beta}+w e_{-\gamma}\right\rangle$. The rank of the invariant quadratic form on such a three-space is 3 for $w \neq 0$ and 1 for $w=0$. In particular it is transverse to the orbit stratification.

\section{Some Gromov-Witten invariants}

In this section we compute explicitly the Gromov-Witten invariants that we need. By Lemma 2.1, these will only be degree one invariants. By Propositions 3.1 and 3.2. the variety of lines in the Cayley Grassmannian is smooth and irreducible of the expected dimension, so we can apply Proposition 2.2 if we use classes of varieties that are transverse to the orbit stratification. We will use either restrictions of Schubert classes from the ambient Grassmannian $G(4,7)$, or when convenient, the classes $\sigma_{8}, \sigma_{7}$ and $\sigma_{6}^{\prime}$ of points, lines and $\beta$-planes in $C G$, which are in general transverse to the orbit stratification (this is obvious for lines, and for planes this is Proposition 3.5.

4.1. The quantum Chevalley formula. The degree one Gromov-Witten invariants that appear in the quantum Chevalley formula are of type $I_{1}\left(\sigma_{1}, \sigma_{k}, \sigma_{\ell}\right)$ for $k+\ell=11$. By the divisor axiom this reduces to the two-points Gromov-Witten invariant $I_{1}\left(\sigma_{k}, \sigma_{\ell}\right)$.

4.1.1. $I_{1}\left(\sigma_{3}, \sigma_{8}\right)$. This invariant is equal to $I_{1}\left(\iota^{*} \tau_{111}, \sigma_{8}\right)$, and can thus be computed as the number of lines passing through a general point $A$, and containing a point $B$ representing a four-space that meets a generic $U_{5} \subset V_{7}$ in dimension at least three. The base of the line is a hyperplane $A_{3}$ of $A$, that meets $U_{5}$ in dimension at least two, since it is also a hyperplane in $B$. But $A_{2}=A \cap U_{5}$ has dimension two, so we need that $A_{2} \subset A_{3} \subset A$. The existence of $B$ is then guaranteed by the fact that the induced map $\wedge^{2} A_{3} \rightarrow\left(U_{5} / A_{2}\right)^{*}$ does not have maximal rank. Generically the rank can drop only by one and $B$ is then uniquely determined. Hence

$$
I_{1}\left(\sigma_{3}^{\prime}, \sigma_{8}\right)=c_{1}\left(\wedge^{2} A_{3}^{*}\right)=2,
$$

where here $A_{3}$ is considered as vector bundle on $\mathbb{P}\left(A / A_{2}\right)=\mathbb{P}^{1}$. 
4.1.2. $I_{1}\left(\sigma_{3}^{\prime}, \sigma_{8}\right)$. This invariant is equal to $I_{1}\left(\iota^{*} \tau_{3}, \sigma_{8}\right)$ and therefore can be computed as the number of lines passing through a general point $A$, and containing a point $B$ representing a four-space that contains a generic $U_{1} \subset V_{7}$. The base of the line is a hyperplane $A_{3}$ of $A$ such that $B=A_{3} \oplus U_{1}$ is in $C G$. The condition for this is that the induced map $\wedge^{2} A_{3} \rightarrow U_{1}^{*}$ is zero. As a consequence

$$
I_{1}\left(\sigma_{3}, \sigma_{8}\right)=c_{3}\left(\wedge^{2} A_{3}^{*}\right)=0,
$$

where $A_{3}$ is considered as vector bundle on $\mathbb{P}(A)=\mathbb{P}^{3}$. The latter Chern class is zero because it is the number of isotropic hyperplanes for a non-degenerate twoforms in four variables.

4.1.3. $I_{1}\left(\iota^{*} \tau_{1111}, \sigma_{7}\right)$. Consider a general line $d$ in $C G$ defined by a pair $V_{3} \subset V_{5}$ representing $\sigma_{7}$ (the same notation will be used for the computations of the next two invariants involving $\sigma_{7}$ ). This invariant is the number of lines in $C G$ meeting $d$, say at $A$, and passing through some $B$ which is contained in a general hyperplane $V_{6}$. The axis of the line is $A_{3} \subset V_{5} \cap V_{6}$. Moreover $A_{3}$ meets $V_{3}$ in codimension one, so necessarily along $V_{3} \cap V_{6}$. Finally, for $A_{3}$ to be contained in some $B \subset V_{6}$ belonging to $C G$ we need that the map $\wedge^{2} A_{3} \rightarrow\left(V_{6} / A_{3}\right)^{*}$ does not have maximal rank. Therefore

$$
I_{1}\left(\iota^{*} \tau_{1111}, \sigma_{7}\right)=c_{1}\left(\wedge^{2} A_{3}^{*}\right)-c_{1}\left(\left(V_{6} / A_{3}\right)=1,\right.
$$

where here $A_{3}$ is considered as vector bundle on $\mathbb{P}\left(V_{5} \cap V_{6} / V_{3} \cap V_{6}\right)=\mathbb{P}^{1}$.

4.1.4. $I_{1}\left(\iota^{*} \tau_{211}, \sigma_{7}\right)$. This invariant is the number of lines in $C G$ meeting $d$ at some $A$, and passing through some $B$ such that $\operatorname{dim}\left(B \cap U_{2}\right) \geq 1$ and $\operatorname{dim}\left(B \cap U_{5}\right) \geq 3$, for $U_{2} \subset U_{5}$ generic. The axis of the line is $A_{3} \subset V_{5}$, and necessarily $\operatorname{dim}\left(A_{3} \cap U_{5}\right) \geq 2$ . We also have $\operatorname{dim}\left(A_{3} \cap V_{3}\right) \geq 2$, and therefore $\operatorname{dim}\left(A_{3} \cap U_{5} \cap V_{3}\right) \geq 1$, which means that $A_{3}$ contains the line $U_{1}=U_{5} \cap V_{3}$. Our parameter space for $A_{3}$ is therefore $\mathbb{P}\left(V_{3} / U_{1}\right) \times \mathbb{P}\left(U_{5} \cap V_{5} / U_{1}\right) \simeq \mathbb{P}^{1} \times \mathbb{P}^{1}$. The condition for the existence of $B$ is that the induced map $\wedge^{2} A_{3} \rightarrow U_{2}^{*}$ does not have full rank. By the Thom-Porteous formula we deduce that

$$
I_{1}\left(\iota^{*} \tau_{211}, \sigma_{7}\right)=c_{2}\left(\wedge^{2} A_{3}^{*}\right)=3 .
$$

Indeed, if $h$ and $h^{\prime}$ are the hyperplane classes on the two copies of $\mathbb{P}^{1}$, then $c\left(A_{3}^{*}\right)=$ $(1+h)\left(1+h^{\prime}\right)$ and $c\left(\wedge^{2} A_{3}^{*}\right)=(1+h)\left(1+h^{\prime}\right)\left(1+h+h^{\prime}\right)$, hence $c_{2}\left(\wedge^{2} A_{3}^{*}\right)=$ $h h^{\prime}+\left(h+h^{\prime}\right)^{2}=3 h h^{\prime}$.

4.1.5. $I_{1}\left(\iota^{*} \tau_{22}, \sigma_{7}\right)$. This is the number of lines in $C G$ meeting $d$ at some $A$, and passing through some $B$ such that $\operatorname{dim}\left(B \cap U_{3}\right) \geq 2$ for $U_{3} \subset V_{7}$ generic. The axis of the line is $A_{3} \subset V_{5}$, and necessarily $\operatorname{dim}\left(A_{3} \cap U_{3}\right) \geq 1$, so $A_{3}$ contains the line $U_{1}=U_{3} \cap V_{5}$. Our parameter space for $A_{3}$ is therefore $\mathbb{P}\left(V_{3}^{*}\right) \simeq \mathbb{P}^{2}$. The condition for the existence of $B$ is that the induced map $\wedge^{2} A_{3} \rightarrow\left(U_{3} / U_{1}\right)^{*}$ does not have full rank. By the Thom-Porteous formula again we deduce that

$$
I_{1}\left(\iota^{*} \tau_{22}, \sigma_{7}\right)=c_{2}\left(\wedge^{2} A_{3}^{*}\right)=2 .
$$

By the restriction formulas 2.3. $\iota^{*} \tau_{1111}=\sigma_{4},, \iota^{*} \tau_{211}=\sigma_{4}+2 \sigma_{4}^{\prime}$ and $\iota^{*} \tau_{22}=$ $\sigma_{4}+\sigma_{4}^{\prime}+\sigma_{4}^{\prime \prime}$, so we deduce that

$$
I_{1}\left(\sigma_{4}, \sigma_{7}\right)=I_{1}\left(\sigma_{4}^{\prime}, \sigma_{7}\right)=1, \quad I_{1}\left(\sigma_{4}^{\prime \prime}, \sigma_{7}\right)=0 .
$$


4.1.6. $I_{1}\left(\iota^{*} \tau_{2111}, \sigma_{6}^{\prime}\right)$. This is the number of lines $d=\left(U_{3}, U_{5}\right)$ in $C G$ meeting

(1) a general $\beta$-plane $P\left(V_{2}, V_{5}\right)$ at a point $A$, such that $V_{2} \subset A \subset V_{5}$,

(2) a general Schubert cycle $\tau_{2111}\left(W_{2}, W_{6}\right)$ at a point $B$, such that $B \cap W_{2} \neq 0$ and $B \subset W_{6}$.

Since $U_{3}$ must be a hyperplane in both $A$ and $B$, we need that $U_{3} \subset V_{4}:=V_{5} \cap W_{6}$. Moreover $U_{3} \cap V_{2}$ must contain a certain one dimensional subspace $V_{1}$. But $U_{3} \cap V_{2} \subset$ $W_{6} \cap V_{2}$, so this must be equal to $V_{1}$. Once $U_{3}$ is fixed, since $U_{3} \cap W_{2} \subset V_{5} \cap W_{2}=0$, there must exist a unique line $L_{1} \subset W_{2}$ such that $B=U_{3}+L_{1}$. Since $A=U_{3}+V_{2}$, the line $d$ is then determined. The set of lines to be considered is thus isomorphic to $\mathbb{P}\left(W_{2}\right) \times \mathbb{P}\left(V_{4} / V_{1}\right)^{*} \simeq \mathbb{P}^{1} \times \mathbb{P}^{2}$.

The condition for $d$ to be contained in $C G$ is that $\Omega\left(U_{3}, U_{3}, L_{1}\right)=0$, which can be interpreted as the vanishing of a general section of the bundle $L_{1}^{*} \otimes \wedge^{2} U_{3}^{*}$. Let us denote by $h_{1}=c_{1}\left(L_{1}^{*}\right)$ and $h_{2}$ the hyperplane classes of our two projective spaces. The Chern roots of $U_{3}^{*}$ are $0, a, b$, with $a+b=h_{2}$ and $a b=h_{2}^{2}$. Then the Chern roots of $L_{1}^{*} \otimes \wedge^{2} U_{3}^{*}$ are $h_{1}+a, h_{1}+b, h_{1}+a+b$ and therefore the invariant we are looking for is

$$
c_{3}\left(L_{1}^{*} \otimes \wedge^{2} U_{3}^{*}\right)=\left(h_{1}+a\right)\left(h_{1}+b\right)\left(h_{1}+a+b\right)=2 h_{1} h_{2}^{2}=2 .
$$

4.1.7. $I_{1}\left(\iota^{*} \tau_{221}, \sigma_{6}^{\prime}\right)$. This is the number of lines $d=\left(U_{3}, U_{5}\right)$ in $C G$ meeting

(1) a general $\beta$-plane $P\left(V_{2}, V_{5}\right)$ at a point $A$, such that $V_{2} \subset A \subset V_{5}$,

(2) a general Schubert cycle $\tau_{221}\left(W_{3}, W_{5}\right)$ at a point $B$, such that $\operatorname{dim}(B \cap$ $\left.W_{3}\right) \geq 2$ and $\operatorname{dim}\left(B \subset W_{5}\right) \geq 3$.

Since $U_{3}$ must be a hyperplane in both $A$ and $B$, we first deduce that $\operatorname{dim}\left(U_{3} \cap V_{2}\right) \geq$ 1 and $\operatorname{dim}\left(U_{3} \cap W_{5}\right) \geq 2$. Since $V_{2} \cap W_{5}=0$, this implies that $U_{3}=L_{1}+P_{2}$ for a line $L_{1} \subset V_{2}$ and a plane $P_{2} \subset V_{3}:=V_{5} \cap W_{5}$. Moreover $P_{2} \cap W_{3} \subset W_{1}:=V_{5} \cap W_{3}$ and since $U_{3} \cap W_{3}$ must be non zero, we need the equality $P_{2} \cap W_{3}=W_{1}$. Once $U_{3}$ is fixed, we need that $B=U_{3}+B_{2}$ with $W_{1} \subset B_{2} \subset W_{3}$ (because of the condition $\left.\operatorname{dim}\left(B \subset W_{3}\right) \geq 2\right)$, and $A=U_{3}+V_{2}$, so the line $d$ is determined.

The set of lines to be considered is thus isomorphic to $\mathbb{P}\left(V_{2}\right) \times \mathbb{P}\left(V_{3} / W_{1}\right) \times$ $\mathbb{P}\left(W_{3} / W_{1}\right) \simeq \mathbb{P}^{1} \times \mathbb{P}^{1} \times \mathbb{P}^{1}$. Let us denote by $h_{1}, h_{2}, h_{3}$ the hyperplane classes of our three projective lines. The condition for $d$ to be contained in $C G$ is that $\Omega\left(U_{3}, U_{3}, B_{2} / W_{1}\right)=0$, which we interprete again as the vanishing of a general section of the bundle $\left(B_{2} / W_{1}\right)^{*} \otimes \wedge^{2} U_{3}^{*}$. The Chern roots of $U_{3}^{*}$ are $0, h_{1}, h_{2}$, hence the invariant we are looking for is

$$
c_{3}\left(\left(B_{2} / W_{1}\right)^{*} \otimes \wedge^{2} U_{3}^{*}\right)=\left(h_{1}+h_{3}\right)\left(h_{2}+h_{3}\right)\left(h_{1}+h_{2}+h_{3}\right)=3 h_{1} h_{2} h_{3}=3 .
$$

By 2.3, $\iota^{*} \tau_{2111}=2 \sigma_{5}$, and $\iota^{*} \tau_{221}=3 \sigma_{5}+\sigma_{5}^{\prime}$, so we deduce that

$$
I_{1}\left(\sigma_{5}, \sigma_{6}^{\prime}\right)=1 \quad \text { and } \quad I_{1}\left(\sigma_{5}^{\prime}, \sigma_{6}^{\prime}\right)=0 .
$$

4.1.8. $I_{1}\left(\iota^{*} \tau_{2111}, \iota^{*} \tau_{2211}\right)$. This is the number of lines in $C G$ joining $A$ and $B$ such that

(1) $A \subset U_{6}$ and $\operatorname{dim}\left(A \cap U_{2}\right) \geq 1$ for some generic $U_{2} \subset U_{6}$,

(2) $B \subset V_{6}$ and $\operatorname{dim}\left(B \cap V_{3}\right) \geq 2$ for some generic $V_{3} \subset V_{6}$.

The axis $D_{3}$ of such a line must be contained in $W_{5}=U_{6} \cap V_{6}$ and meet $V_{3}$ non trivially, along a line $D_{1} \subset W_{2}=U_{6} \cap V_{3}$. The parameter space $P$ for the pair $D_{1} \subset D_{3}$ is the quadric bundle $G\left(2, W_{5} / D_{1}\right)$ over $\mathbb{P}\left(W_{2}\right) \simeq \mathbb{P}^{1}$. We need that the three-form $\Omega$ vanish on $D_{3}$, a codimension one condition. If $D_{3}$ does not contain $W_{2}$ or $W_{1}=U_{2} \cap V_{6}$, for $A$ and $B$ to exist we need that the induced maps $\wedge^{2} D_{3} \rightarrow U_{2}^{*}$ 
and $\wedge^{2} D_{3} \rightarrow\left(V_{3} / D_{1}\right)^{*}$ do not have maximal ranks, each of which is a codimension two condition. The number of points satisfying these conditions is given by:

$$
c_{1}\left(D_{3}^{*}\right) c_{2}\left(\wedge^{2} D_{3}^{*}\right) c_{2}\left(\wedge^{2} D_{3}^{*}-\left(V_{3} / D_{1}\right)\right)=7 .
$$

Indeed, this intersection product has to be taken in $P$, whose cohomology ring is generated by the hyperplane class $h=c_{1}\left(D_{1}^{*}\right)$ pulled-back from $\mathbb{P}^{1}$, and the Chern classes $a_{1}, a_{2}$ of the dual tautological vector bundle $\left(D_{3} / D_{1}\right)^{*}$. We leave the details of the computation to the reader.

However, the important point to notice is that among the seven points $p_{1}, \cdots, p_{7}$ at which our previous conditions are satisfied, some may not correspond to an actual line inside $C G$ passing through $A$ and $B$. Indeed, it could happen that $W_{2} \subset D_{3}$ or $W_{1} \subset D_{3}$. Let us study these two cases separately.

Consider the case where $W_{2} \subset D_{3}$. The parameter space for $D_{3}$ is then $\mathbb{P}\left(W_{5} / W_{2}\right)$. Among $p_{1}, \cdots, p_{7}$, the points that satisfy $W_{2} \subset D_{3}$ are those for which $\left.\omega\right|_{D_{3}}=0$ (a codimension one condition) and the induced map $\wedge^{2} D_{3} \rightarrow U_{2}^{*}$ does not have maximal rank (a codimension two condition). Indeed, notice that $W_{2} \subset D_{3}$ implies automatically that $\wedge^{2} D_{3} \rightarrow\left(V_{3} / D_{1}\right)^{*}$ does not have maximal rank. As $\operatorname{dim}\left(\mathbb{P}\left(W_{5} / W_{2}\right)\right)=2$, these conditions will not be satisfied generically.

Consider then the case $W_{1} \subset D_{3}$. The parameter space for $D_{1} \subset D_{3}$ is now the projective bundle $\mathbb{P}\left(W_{5} /\left(W_{1}+D_{1}\right)\right)$ over $\mathbb{P}\left(W_{2}\right)$. Among $p_{1}, \cdots, p_{7}$, the points that satisfy $W_{1} \subset D_{3}$ are those for which $\left.\omega\right|_{D_{3}}=0$ (codimension one) and the induced map $\wedge^{2} D_{3} \rightarrow\left(V_{3} / D_{1}\right)^{*}$ does not have maximal rank (codimension two). Their number is $c_{1}\left(D_{3}^{*}\right) c_{2}\left(\wedge^{2} D_{3}^{*}-\left(V_{3} / D_{1}\right)\right)=1$.

As a consequence, we obtain:

$$
I_{1}\left(\iota^{*} \tau_{2111}, \iota^{*} \tau_{2211}\right)=2 I_{1}\left(\sigma_{5}, \sigma_{6}\right)+6 I_{1}\left(\sigma_{5}, \sigma_{6}^{\prime}\right)=7-1=6 .
$$

4.1.9. $I_{1}\left(\iota^{*} \tau_{32}, \iota^{*} \tau_{2211}\right)$. This is the number of lines in $C G$ joinning $A$ and $B$ such that

(1) $U_{1} \subset A$ and $\operatorname{dim}\left(A \cap U_{3}\right) \geq 2$ for some generic $U_{1} \subset U_{3}$,

(2) $B \subset V_{6}$ and $\operatorname{dim}\left(B \cap V_{3}\right) \geq 2$ for some generic $V_{3} \subset V_{6}$.

The axis $D_{3}$ of such a line must be contained in $V_{6}$; moreover it must satisfy $D_{3} \cap U_{1} \subset U_{1} \cap V_{6}=0, \operatorname{dim}\left(D_{3} \cap U_{3}\right) \geq 1$ and $\operatorname{dim}\left(D_{3} \cap V_{3}\right) \geq 1$. The last two conditions imply the existence of two lines $D_{1}$ and $D_{1}^{\prime}$ inside $D_{3}$ which are contained respectively in $V_{6} \cap U_{3}=W_{2}$ and $V_{3}$. The parameter space $P$ for $D_{1}, D_{1}^{\prime} \subset D_{3}$ is the projective bundle $\mathbb{P}\left(V_{6} /\left(D_{1}+D_{1}^{\prime}\right)\right)$ over $\mathbb{P}\left(W_{2}\right) \times \mathbb{P}\left(V_{3}\right)$. We need that the three-form $\omega$ vanishes on $D_{3}$, a codimension one condition. If $D_{3} \cap V_{3}=D_{1}^{\prime}$, for $A$ and $B$ to exist we need that the induced maps $\wedge^{2} D_{3} \rightarrow U_{1}^{*}$ and $\wedge^{2} D_{3} \rightarrow\left(V_{3} / D_{1}^{\prime}\right)^{*}$ do not have maximal ranks, the first being a codimension three and the second a codimension two condition. The number of points satisfying these conditions is given by:

$$
c_{1}\left(D_{3}^{*}\right) c_{3}\left(\wedge^{2} D_{3}^{*}\right) c_{2}\left(\wedge^{2} D_{3}^{*}-\left(V_{3} / D_{1}^{\prime}\right)\right)=4,
$$

this intersection product being taken in $P$. One can easily verify that none of these four points satisfy $\operatorname{dim}\left(D_{3} \cap V_{3}\right) \geq 2$. As a consequence, we obtain:

$$
I_{1}\left(\iota^{*} \tau_{32}, \iota^{*} \tau_{2211}\right)=I_{1}\left(\sigma_{5}, \sigma_{6}\right)+3 I_{1}\left(\sigma_{5}, \sigma_{6}^{\prime}\right)+I_{1}\left(\sigma_{5}^{\prime}, \sigma_{6}\right)+3 I_{1}\left(\sigma_{5}^{\prime}, \sigma_{6}^{\prime}\right)=4 .
$$

This is enough to deduce: 
Proposition 4.1. Up to degree three the quantum product with the hyperplane class is equal to the classical product. Up to degree seven, it is given by:

$$
\begin{array}{llc}
\sigma_{3} \sigma_{1} & = & 2 \sigma_{4}+2 \sigma_{4}^{\prime}+2 q, \\
\sigma_{3}^{\prime} \sigma_{1}= & \sigma_{4}^{\prime}+\sigma_{4}^{\prime \prime}, \\
\sigma_{4} \sigma_{1}= & 2 \sigma_{5}+q \sigma_{1}, \\
\sigma_{4}^{\prime} \sigma_{1}= & 2 \sigma_{5}+\sigma_{5}^{\prime}+q \sigma_{1}, \\
\sigma_{4}^{\prime \prime} \sigma_{1}= & \sigma_{5}^{\prime}, \\
\sigma_{5} \sigma_{1}= & \sigma_{6}+2 \sigma_{6}^{\prime}+q \sigma_{2}^{\prime}, \\
\sigma_{5}^{\prime} \sigma_{1}= & 3 \sigma_{6}+2 \sigma_{6}^{\prime}+q \sigma_{2}, \\
\sigma_{6} \sigma_{1}= & \sigma_{7}+q \sigma_{3}^{\prime}, \\
\sigma_{6}^{\prime} \sigma_{1}= & \sigma_{7}+q \sigma_{3} .
\end{array}
$$

4.2. The missing invariants. Recall that in order to determine completely the quantum multiplication, we just need to determine the products $\sigma_{2}^{2}$ and $\sigma_{4} \sigma_{2}$. This requires the computation of three more Gromov-Witten invariants.

4.2.1. $I_{1}\left(\sigma_{2}, \sigma_{2}, \sigma_{8}\right)$. This invariant is $I_{1}\left(\iota^{*} \tau_{2}, \iota^{*} \tau_{2}, \sigma_{8}\right)$. It can therefore be computed as the number of lines $d=\left(D_{3}, D_{5}\right)$ in $C G$ joining three points $A, B, C$ such that $A$ (resp. $B$ ) meets non trivially a general $A_{2}$ (resp. $B_{2}$ ), and $C$ is a general point in $C G$. Since $C \cap B_{2}=0$, we must have $D_{5} \subset C+B_{2}$, hence $A \cap A_{2} \subset\left(C+B_{2}\right) \cap A_{2}$, which is a line $A_{1}$, and there must be equality. Symmetrically $B \cap B_{2}=B_{1}:=\left(C+A_{2}\right) \cap B_{2}$. The line $d$ is therefore determined by $U_{3} \subset C$, which gives $A=D_{3}+A_{1}$ and $B=D_{3}+B_{1}$.

The condition that $d$ is contained in $C G$ reduces to $\Omega\left(D_{3}, D_{3}, A_{1}\right)=0$. So the invariant we are looking for is computed on $\mathbb{P}\left(C^{*}\right)$ as

$$
I_{1}\left(\sigma_{2}, \sigma_{2}, \sigma_{8}\right)=c_{3}\left(\wedge^{2} D_{3}^{*}\right)=0 .
$$

4.2.2. $I_{1}\left(\sigma_{2}, \sigma_{4}, \sigma_{6}^{\prime}\right)$. We compute this Gromov-Witten invariant as $I_{1}\left(\iota^{*} \tau_{2}, \iota^{*} \tau_{1111}, \sigma_{6}^{\prime}\right)$, so as the number of lines in $C G$ joining three points $A, B, C$ such that

(1) $A$ meets non trivially a general $U_{2}$,

(2) $B$ is contained in a general hyperplace $H_{6}$,

(3) $C$ belongs to a general $\beta$-plane defined by a pair $V_{2} \subset V_{5}$.

The axis $D_{3}$ of the line is contained in $A, B, C$, hence in $V_{5} \cap H_{6}$. Moreover it must meet $V_{2}$ in dimension at least one, so necessarily along $V_{2} \cap H_{6}$. Thus $V_{2} \cap H_{6} \subset$ $D_{3} \subset V_{5} \cap H_{6}$ and $D_{3}$ is parametrized by a $\mathbb{P}^{2}$. Then to get $A$ we need the induced map $\wedge^{2} D_{3} \rightarrow U_{2}^{*}$ not to be of maximal rank. Necessarily $C=D_{3}+V_{2}$. Since $D_{3} \subset H_{6}$ there is a unique $B$ on the line joining $A$ to $C$ which is contained in $H_{6}$. It is automatically in $C G$ since $A$ and $C$ are. We conclude that

$$
I_{1}\left(\sigma_{2}, \sigma_{4}, \sigma_{6}^{\prime}\right)=c_{2}\left(\wedge^{2} D_{3}^{*}\right)=2 .
$$

4.2.3. $I_{1}\left(\sigma_{2}, \sigma_{4}, \sigma_{6}\right)$. Let us compute $I_{1}\left(\iota^{*} \tau_{1111}, \iota^{*} \tau_{2}, \iota^{*} \tau_{33}\right)=I_{1}\left(\sigma_{4}, \sigma_{2}, \sigma_{6}+\sigma_{6}^{\prime}\right)$. This is the number of lines $d=\left(D_{3}, D_{5}\right)$ in $C G$ joining three points $A, B, C$ such that

(1) $A$ is contained in a general $A_{6}$,

(2) $B$ meets non trivially a general $B_{2}$,

(3) $C$ contains a general $C_{2}$.

The axis $D_{3}$ must then be contained in $A_{6}$, and meet $C_{2}$ along a line, necessarily $C_{1}:=C_{2} \cap A_{6}$. Then $C$ must be $D_{3}+C_{2}$ and $B$ must be generated by $D_{3}$ and a line $B_{1}$ of $B_{2}$. (Beware that potentially the intersection $B_{1}=B \cap B_{2}$ could 
be contained in $D_{3}$, which would impose $B_{1}=A_{6} \cap B_{2}$. But then the isotropy conditions would include $\Omega\left(B_{1}, C_{2}, C_{2}\right)=0$, which is not possible.) We then get $D_{5}=D_{3}+C_{2}+B_{1}$ and $A=D_{5} \cap A_{6}$. We are thus led to consider a set of lines parametrized by $\mathbb{P}\left(B_{2}\right) \times G\left(2, A_{6} / C_{1}\right)=\mathbb{P}^{1} \times G(2,5)$.

The isotropy conditions are $\Omega\left(D_{3}, D_{3}, C_{1}\right)=0, \Omega\left(D_{3}, D_{3}, C_{2} / C_{1}\right)=0$ and $\Omega\left(D_{3}, D_{3}, B_{1}\right)=0$. The bundle $D_{3} / C_{1}$ is just the tautological bundle on the Grassmannian, let us denote the Chern roots of its dual by $a, b$ with $a+b=\tau_{1}$, $a b=\tau_{11}$ the usual Schubert classes. Let $h$ be the hyperplane class on $\mathbb{P}\left(B_{2}\right)$. Our invariant is equal to $c_{1}\left(\wedge^{2}\left(D_{3} / C_{1}\right)^{*}\right) c_{3}\left(\wedge^{2} D_{3}^{*}\right) c_{3}\left(B_{1}^{*} \otimes \wedge^{2} D_{3}^{*}\right)$, that is,

$$
\tau_{1} a b(a+b)(a+h)(b+h)(a+b+h)=\tau_{1}^{2} \tau_{11}\left(\tau_{11}+\tau_{1}^{2}\right) h=3 .
$$

This gives three points that satisfy the conditions $\Omega\left(D_{3}, D_{3}, C_{1}\right)=0$,

$\Omega\left(D_{3}, D_{3}, C_{2} / C_{1}\right)=0$ and $\Omega\left(D_{3}, D_{3}, B_{1}\right)=0$ over $\mathbb{P}\left(B_{2}\right) \times G\left(2, A_{6} / C_{1}\right)$. Among them, we still need to remove those for which $B=C$. As we already know that $C_{1} \subset D_{3}$, we have $B=C$ if $\operatorname{dim}\left(D_{3} \cap W_{3}\right) \geq 2$, where $W_{3}=A_{6} \cap\left(B_{2}+C_{2}\right)$. Let $C_{1} \subset D_{2} \subset\left(D_{3} \cap W_{3}\right)$ with $\operatorname{dim}\left(D_{2}\right)=2$. Then the parameter space of $D_{2} \subset D_{3}$ is the projective bundle $\mathbb{P}\left(A_{6} / D_{2}\right)$ over $\mathbb{P}\left(W_{3} / C_{1}\right)$, and the conditions we need to impose are $\Omega\left(D_{3}, D_{3}, C_{1}\right)=0$ and $\Omega\left(D_{3}, D_{3}, C_{2} / C_{1}\right)=0$. Let $l$ be the hyperplane in $\mathbb{P}\left(W_{3} / C_{1}\right) \cong \mathbb{P}^{1}$, and $m$ the relative hyperplane class in $\mathbb{P}\left(A_{6} / D_{2}\right)$. The number of points that we need to remove is therefore

$$
c_{1}\left(\wedge^{3} D_{3}^{*}\right) c_{3}\left(\wedge^{2} D_{3}^{*}\right)=(l+m) \operatorname{lm}(l+m)=l m^{3}=1,
$$

and we get

$$
I_{1}\left(\sigma_{4}, \sigma_{2}, \sigma_{6}\right)=3-1-I_{1}\left(\sigma_{2}, \sigma_{4}, \sigma_{6}^{\prime}\right)=0 .
$$

This finally yields the two missing products:

$$
\sigma_{2}^{2}=\sigma_{4}+2 \sigma_{4}^{\prime}+2 \sigma_{4}^{\prime \prime}, \quad \sigma_{4} \sigma_{2}=\sigma_{6}+\sigma_{6}^{\prime}+2 q \sigma_{2}^{\prime} .
$$

4.3. A presentation of the quantum cohomology ring. We now have enough information to deduce a presentation of the quantum cohomology ring. We first use the relations we have obtained so far to express the Schubert classes in terms of the generators $\sigma_{1}$ and $\sigma_{2}$. We could also have chosen the other degree two class $\sigma_{2}^{\prime}=\sigma_{1}^{2}-\sigma_{2}$ but the formulas would be slightly worse. In degree three there is no quantum corrections, we easily get

$$
\sigma_{3}=\frac{1}{4}\left(3 \sigma_{1}^{3}-5 \sigma_{1} \sigma_{2}\right), \quad \sigma_{3}^{\prime}=\frac{1}{4}\left(3 \sigma_{1} \sigma_{2}-\sigma_{1}^{3}\right) .
$$

In degree four knowing $\sigma_{3} \sigma_{1}, \sigma_{3}^{\prime} \sigma_{1}$, and $\sigma_{2}^{2}$ that we have just computed, we deduce:

$$
\begin{aligned}
\sigma_{4} & =\sigma_{2}^{2}-\frac{3}{2} \sigma_{2} \sigma_{1}^{2}+\frac{1}{2} \sigma_{1}^{4}, \\
\sigma_{4}^{\prime} & =-\sigma_{2}^{2}+\frac{7}{8} \sigma_{2} \sigma_{1}^{2}-\frac{1}{8} \sigma_{1}^{4}-q, \\
\sigma_{4}^{\prime \prime} & =\sigma_{2}^{2}-\frac{1}{8} \sigma_{2} \sigma_{1}^{2}-\frac{1}{8} \sigma_{1}^{4}+q .
\end{aligned}
$$

The quantum products of these classes by the hyperplane class will give not only an expression of $\sigma_{5}$ and $\sigma_{5}^{\prime}$ in terms of the generators, but also a degree five relation 
in the quantum cohomology ring. We get

$$
\begin{aligned}
\sigma_{5} & =\frac{1}{2} \sigma_{2} \sigma_{1}^{3}-\sigma_{2}^{2} \sigma_{1}-\frac{3}{2} q \sigma_{1}, \\
\sigma_{5}^{\prime} & =-\frac{3}{4} \sigma_{2} \sigma_{1}^{3}+\frac{7}{4} \sigma_{2}^{2} \sigma_{1}+\frac{3}{2} q \sigma_{1},
\end{aligned}
$$

plus the degree five relation $\left(\sigma_{4}^{\prime}-\sigma_{4}-\sigma_{4}^{\prime \prime}\right) \sigma_{1}=0$. In degree six, we directly get $\sigma_{6}$ and $\sigma_{6}^{\prime}$ from $\sigma_{5} \sigma_{1}$ and $\sigma_{5}^{\prime} \sigma_{1}$ :

$$
\begin{aligned}
\sigma_{6} & =-\frac{5}{8} \sigma_{2} \sigma_{1}^{4}+\frac{11}{8} \sigma_{2}^{2} \sigma_{1}^{2}+q\left(2 \sigma_{1}^{2}-\sigma_{2}\right), \\
\sigma_{6}^{\prime} & =\frac{9}{16} \sigma_{2} \sigma_{1}^{4}-\frac{19}{16} \sigma_{2}^{2} \sigma_{1}^{2}+q\left(\sigma_{2}-\frac{9}{4} \sigma_{1}^{2}\right) .
\end{aligned}
$$

Our computation of $\sigma_{4} \sigma_{2}$ yields a degree 6 relation and we get:

Proposition 4.2. The rational quantum cohomology ring of the Cayley Grassmannian is $Q H^{*}(C G, \mathbb{Q})=\mathbb{Q}\left[\sigma_{1}, \sigma_{2}, q\right] /\left\langle R_{5}(q), R_{6}(q)\right\rangle$, for the quantum relations

$$
\begin{aligned}
& R_{5}(q)=\sigma_{1}^{5}-5 \sigma_{1}^{3} \sigma_{2}+6 \sigma_{1} \sigma_{2}^{2}+4 q \sigma_{1}, \\
& R_{6}(q)=16 \sigma_{2}^{3}-27 \sigma_{1}^{2} \sigma_{2}^{2}+9 \sigma_{1}^{4} \sigma_{2}+32 q \sigma_{2}-28 q \sigma_{1}^{2} .
\end{aligned}
$$

A routine computation allows to check that for $q \neq 0$, these two equations define a reduced scheme.

Corollary 4.3. The quantum cohomology ring $\left.Q H^{*}(C G, \mathbb{Q})\right|_{q=1}$ is semisimple.

4.4. Completing the Chevalley and Giambelli formulas. We have enough information to complete the quantum Chevalley formula up to degree seven. The quantum product $\sigma_{7} \sigma_{1}$ yields $\sigma_{8}$ up to a potential term in $q^{2}$. Plugging this into the product $\sigma_{8} \sigma_{1}$, we conclude that this term is in fact zero. This allows to complete the quantum Giambelli formula by the two equations

$$
\begin{aligned}
\sigma_{7} & =\frac{1}{18} \sigma_{2}^{3} \sigma_{1}+q\left(\frac{13}{36} \sigma_{1} \sigma_{2}-\frac{17}{36} \sigma_{1}^{3}\right), \\
\sigma_{8} & =\frac{1}{9} \sigma_{2}^{4}+q\left(\frac{2}{9} \sigma_{2}^{2}+\frac{29}{36} \sigma_{1}^{2} \sigma_{2}-\frac{27}{36} \sigma_{1}^{4}\right)+q^{2} .
\end{aligned}
$$

Finally, the missing products in the quantum Chevalley formula are

$$
\sigma_{7} \sigma_{1}=\sigma_{8}+q \sigma_{4}+q \sigma_{4}^{\prime}, \quad \sigma_{8} \sigma_{1}=2 q \sigma_{5} .
$$

From that, it is straightforward to deduce the full multiplication table, that we report in the Appendix. Remarkably, all the coefficients are non negative.

The mere fact that the quantum product by the hyperplane class has only non negative coefficients allows to ensure, following the approach of [CL], that conjecture $\mathcal{O}$ from GGI is verified in this case:

Corollary 4.4. The Cayley Grassmannian satisfies conjecture $\mathcal{O}$.

The eigenvalues of the quantum product by the hyperplane class are the roots of the polynomial

$$
p(t)=-t^{15}+102 t^{11} q-317 t^{7} q^{2}+2048 t^{3} q^{3}=t^{3} f\left(t^{4}\right),
$$

where $f(y)=-y^{3}+102 y^{2} q-317 y q^{2}+2048 q^{3}$. The equation $f(y)=0$ has three distinct solutions, among which the one with maximal modulus is real, namely 
$y_{\max } \simeq 99.00713881372502$. As far as the anticanonical class $-K_{C G}=4 \sigma_{1}$ is concerned, we deduce that its spectral radius is

$$
T(C G)=4\left(y_{\max }\right)^{1 / 4} \simeq 12.6175960332>\operatorname{dim} C G+1,
$$

in agreement with a conjecture of Galkin Ga].

\section{ON THE DERIVED CATEGORY OF $C G$}

Denote by $T$ and $Q$ the ranks four and three tautological bundles of the Grassmannian $G$, and their restrictions to $C G$ as well.

5.1. An exceptional collection. According to Dubrovin's conjecture, the semisimplicity of the cohomology ring of $C G$ should imply that the bounded derived category of sheaves $D^{b}(C G)$ admits a full exceptional collection. The length of this collection should be equal to the rang of the Grothendieck group of vector bundles on $C G$, which is equal to 15 . We have been able to find an exceptional collection of this length, as follows.

Consider the collections:

$$
\begin{aligned}
& \mathcal{C}_{0}=\left\langle\mathfrak{s l}(Q), \mathcal{O}_{G}, Q, \wedge^{2} T^{\vee}, \wedge^{2} Q\right\rangle \\
& \mathcal{C}_{1}=\left\langle\mathcal{O}_{G}, Q, \wedge^{2} T^{\vee}, \wedge^{2} Q\right\rangle \\
& \mathcal{C}_{2}=\left\langle\mathcal{O}_{G}, Q, \wedge^{2} Q\right\rangle \\
& \mathcal{C}_{3}=\mathcal{C}_{2}
\end{aligned}
$$

Proposition 5.1. $\mathcal{C}=\left\langle\mathcal{C}_{0}, \mathcal{C}_{1}(1), \mathcal{C}_{2}(2), \mathcal{C}_{3}(3)\right\rangle$ is a Lefschetz exceptional collection inside the bounded category of sheaves on $C G$.

Here "Lefschetz" refers to the particular structure of the collection [Ku1]: we have $\mathcal{C}_{0} \supset \mathcal{C}_{1} \supset \mathcal{C}_{2} \supset \mathcal{C}_{3}$ and $\mathcal{C}_{i}(i)$ is the result of twisting the objects in $\mathcal{C}_{i}$ by $\mathcal{O}_{C G}(i)$.

Of course we expect $\mathcal{C}$ to be full, in the sense that it should generate the full derived category. In order to prove such a statement, it can be useful to have a good birational model of the variety under consideration. We will end this paper by providing such a simple model.

5.2. A birationality. Consider a decomposition $V_{7}=V_{6} \oplus V_{1}$. For any $k$, there is an induced projection $\pi_{k}: G\left(k, V_{7}\right) \rightarrow G\left(k, V_{6}\right)$. The exceptional locus of this rational map is the sub-Grassmannian of $k$-planes containing $V_{1}$, which is isomorphic to $G\left(k-1, V_{6}\right)$. Let $\tilde{G}\left(k, V_{7}\right)$ be the variety of pairs $\left(V_{k}, U_{k}\right)$ of $k$-planes with $U_{k} \subset V_{6}$ and $V_{k} \subset U_{k} \oplus V_{1} \subset V_{7}$. The two projections induce a diagram

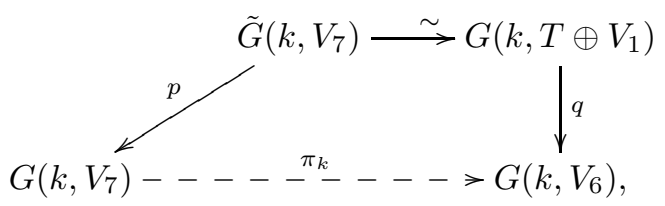

where $p$ is the blow-up of $G\left(k-1, V_{6}\right)$ and $q$ is a relative Grassmannian.

Recall that $C G$ is defined by a three-form $\Omega$ on $V_{7}$. We let $\omega$ denote its restriction to $V_{6}$. Moreover, we can fix a generator $e$ of $V_{1}$, and get on $V_{6}$ the two-form $\alpha=\Omega(e, \bullet, \bullet)$. For a general choice of $V_{1}$, this is a non degenerate two-form. The following result is Proposition 6.3 in [Ku3]. 
Proposition 5.2. The projection $\pi_{2}$ sends the adjoint variety $G_{2}^{\text {ad }}$ to its image $Y_{2} \subset I G_{\alpha}(2,6) \subset G\left(2, V_{6}\right)$.

Proof. The intersection of $G_{2}^{a d}$ with the exceptional locus of $\pi_{2}$ is the variety of planes of the form $V_{2}=U_{1} \oplus V_{1}$ for which $\Omega\left(v_{2}, v_{2}^{\prime}, \bullet\right)=0$ for any basis $v_{2}, v_{2}^{\prime}$. Choosing $v_{2}^{\prime}=e$, this amounts to $\alpha\left(v_{2}, \bullet\right)=0$. Equivalently, $v_{2}$ would belong to the kernel of $\alpha$; but this kernel is zero, so the restriction of $\pi_{2}$ to $G_{2}^{a d}$ is well-defined.

So any point of $G_{2}^{a d}$ is of the form $V_{2}=U_{2}^{\phi}=\left\{x+\phi(x), x \in U_{2}\right\}$ for some $U_{2} \subset V_{6}$ and $\phi \in \operatorname{Hom}\left(U_{2}, V_{1}\right)$. The condition for $V_{2}$ to belong to $G_{2}^{a d}$ is that $U_{2}=\left\langle u, u^{\prime}\right\rangle$ is $\alpha$-isotropic and

$$
\omega\left(u, u^{\prime}, v\right)+\phi(u) \alpha\left(u^{\prime}, v\right)-\phi\left(u^{\prime}\right) \alpha(u, v)=0 \quad \bmod U_{2}
$$

for any $v \in V_{6}$. In particular, since the linear forms $\alpha(u, \bullet)$ and $\alpha\left(u^{\prime}, \bullet\right)$ are linearly independent, $\phi$ is uniquely determined. So $G_{2}^{a d}$ is projected bijectively onto its image $Y$ in $G\left(2, V_{6}\right)$.

By orthogonality with respect to $\alpha, I G_{\alpha}(2,6)$ is mapped isomorphically to the variety of coisotropic 4-planes, those 4-planes on which $\alpha$ has rank two.(in other words, they contain their orthogonal 2-plane). Moreover, the image of $Y_{2}$ in $G\left(4, V_{6}\right)$ can be interpreted as the variety $Y_{4}$ of pairs $\left(U_{2} \subset U_{4}=U_{2}^{\perp}\right)$ such that $\omega\left(U_{2}, U_{2}, U_{4}\right)=0$.

Proposition 5.3. The projection $\pi_{4}$ maps the Cayley Grassmannian CG birationally onto $G\left(4, V_{6}\right)$. This birational map is resolved by blowing up a hyperplane section of the Lagrangian Grassmannian $Z_{3}=L G_{\alpha}(3,6) \cap H_{\omega}$, which yields an isomorphism with the blowup of $G\left(4, V_{6}\right)$ along $Y_{4}$.

Proof. The intersection of $C G$ with the exceptional locus of $\pi_{4}$ is the variety of 4planes of the form $V_{4}=U_{3} \oplus V_{1}$ on which $\Omega$ vanishes identically. Equivalently, $\omega$ and $\alpha$ need to vanish on $U_{3}$, or in other words $U_{3}$ has to belong to $Z_{3}=L G_{\alpha}(3,6) \cap H_{\omega}$. The preimage of such a $V_{4}$ in $\tilde{G}\left(4, V_{7}\right)$ is then isomorphic to the variety of 4-planes $U_{4}$ such that $U_{3} \subset U_{4} \subset V_{6}$, which yields a projective plane. Restricting the previous diagram, we get

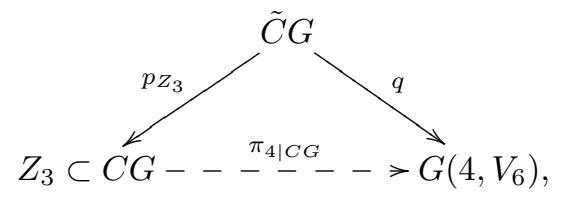

where $p_{Z_{3}}$ is the blow-up of $Z_{3}$.

Let us check that the projection $\pi_{4}$ restricted to $C G$ is birational. The preimage of a 4-plane $U_{4} \subset V_{6}$ is the set of 4-planes $V_{4} \subset V_{7}$ of the form $V_{4}=U_{4}^{\phi}$, for $\phi \in$ $\operatorname{Hom}\left(U_{4}, V_{1}\right)$. The condition that $\Omega$ vanishes on $V_{4}$ is equivalent to the condition that $\omega+\phi \wedge \alpha=0$ on $U_{4}$. When $\alpha_{\mid U_{4}}$ has rank four, the wedge product by this two-form yields an isomorphism from $U_{4}^{\vee}$ to $\wedge^{3} U_{4}^{\vee}$, so that $\phi$ is determined uniquely.

This also implies that $q$ has non-trivial fibers only over the locus of those $U_{4}$ 's on which $\alpha$ has rank two, which means that $U_{4}$ is coisotropic; let $U_{2}=U_{4}^{\perp}$. Suppose that $V_{4} \subset U_{4} \oplus V_{1}$ belongs to $C G$. If $V_{4}=U_{3} \oplus V_{1}$, we need that $\omega$ and $\alpha$ vanish on $U_{3}$, and the latter condition means that $U_{2} \subset U_{3} \subset U_{4}$. If $V_{4}$ is of the form $U_{4}^{\phi}$ with $\omega+\phi \wedge \alpha=0$ on $U_{4}$, then clearly $\omega\left(U_{2}, U_{2}, U_{4}\right)=0$, and conversely this condition implies the existence of a suitable $\phi$. So the exceptional locus $Y$ of $q^{-1}$ is isomorphic to $G_{2}^{a d}$. Moreover, $\phi$ is determined in $V_{4}^{\vee}$ only up to $U_{2}^{\perp}$, which means 
that the fiber contains an affine linear open subset of dimension two; so the fiber, being the closure of this affine linear subspace, is in fact a projective plane. This implies that the projection $q$ is in fact the blowup of $Y_{4}$ in $G\left(4, V_{6}\right)$.

We get the following diagram

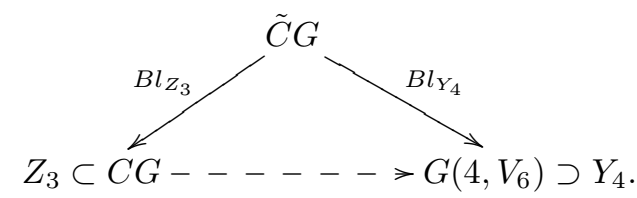

Note that $Z_{3}$ and $Y_{4} \simeq G_{2}^{a d}$ are two examples of five dimensional minifolds GKMS: their derived categories admit full exceptional collections of length 6 .

Indeed Kuznetsov proved in [Ku1, 6.4] that the derived category of the adjoint variety $G_{2}^{a d}$ has a Lefschetz decomposition

$$
D\left(G_{2}^{a d}\right)=\left\langle\mathcal{Y}_{0}, \mathcal{Y}_{1}(1), \mathcal{Y}_{2}(2)\right\rangle
$$

where $\mathcal{Y}_{0}=\mathcal{Y}_{1}=\mathcal{Y}_{2}=\left\langle\mathcal{O}, T^{\vee}\right\rangle$, the bundle $T$ being the restriction of the rank two tautological bundle from the Grassmannian $G(2,7)$.

Moreover, a completely similar statement holds for a smooth hyperplane section $Z_{3}$ of the Lagrangian Grassmannian $L G(3,6)$ : according to $\mathrm{Sa}$,

$$
D\left(Z_{3}\right)=\left\langle\mathcal{Z}_{0}, \mathcal{Z}_{1}(1), \mathcal{Z}_{2}(2)\right\rangle
$$

where $\mathcal{Z}_{0}=\mathcal{Z}_{1}=\mathcal{Z}_{2}=\left\langle\mathcal{O}, U^{\vee}\right\rangle$, the bundle $U$ being the restriction of the rank three tautological bundle from the Grassmannian $G(3,6)$.

For the derived category of the Grassmannian $G(2,6)$, several full exceptional collections are known: the two Kapranov collections, and also the minimal Lefschetz collection found by Kuznetsov Ku2]:

$$
D(G(2,6))=\left\langle\mathcal{A}_{0}, \mathcal{A}_{1}(1), \mathcal{A}_{2}(2), \mathcal{A}_{3}(3), \mathcal{A}_{4}(4), \mathcal{A}_{5}(5)\right\rangle
$$

where $\mathcal{A}_{0}=\mathcal{A}_{1}=\mathcal{A}_{2}=\left\langle\mathcal{O}, T^{\vee}, S^{2} T^{\vee}\right\rangle$ and $\mathcal{A}_{3}=\mathcal{A}_{4}=\mathcal{A}_{5}=\left\langle\mathcal{O}, T^{\vee}\right\rangle$. This Lefschetz collection induces a minimal Lefschetz collection on $I G(2,6) \mathrm{Ku} 2$.

Acknowledgements. We warmly thank Alexander Kuznetsov for useful comments.

\section{REFERENCES}

[CL] Cheong D., Li C., On the conjecture $\mathcal{O}$ of GGI for G/P. Adv. Math. 306 (2017), 704-721.

[Ga] Galkin S., The conifold point, arXiv:1404.7388

[GKMS] Galkin S., Katzarkov L., Mellit A., Shinder E., Minifolds and Phantoms, arXiv: 1305.4549

[GPPS] Gonzales R., Pech C., Perrin N., Samokhin A., Geometry of horospherical varieties of Picard rank one, arXiv:1803.05063.

[GGI] Galkin S., Golyshev V., Iritani H., Gamma classes and quantum cohomology of Fano manifolds: gamma conjectures, Duke Math. J. 165 (2016), 2005-2077.

[Ku1] Kuznetsov A., Hyperplane sections and derived categories, Izv. Math. 70 (2006), 447-547.

[Ku2] Kuznetsov A., Exceptional collections for Grassmannians of isotropic lines, Proc. Lond. Math. Soc. 97 (2008), 155-182.

[Ku3] Kuznetsov A., Küchle fivefolds of type c5, Math. Z. 284 (2016), 1245-1278.

[Ma] Manivel L., The Cayley Grassmannian, J. Algebra 503 (2018), 277-298.

[Sa] Samokhin A., On the derived category of coherent sheaves on a 5-dimensional Fano variety, C. R. Math. Acad. Sci. Paris 340 (2005), 889-893.

[TS] Siebert B., Tian G., On quantum cohomology rings of Fano manifolds and a formula of Vafa and Intriligator, Asian J. Math. 1 (1997), 679-695. 


\section{Appendix: The Quantum multiplication table}

Here is the complete quantum multiplication table of the Cayley Grassmannian:

$$
\begin{aligned}
& \sigma_{1} \sigma_{1}=\sigma_{2}+\sigma_{2}^{\prime}, \quad \sigma_{4}^{\prime \prime} \sigma_{1}=\sigma_{5}^{\prime}, \\
& \sigma_{2} \sigma_{1}=\sigma_{3}+3 \sigma_{3}^{\prime}, \quad \sigma_{5} \sigma_{1}=\sigma_{6}+2 \sigma_{6}^{\prime}+q \sigma_{2}^{\prime}, \\
& \sigma_{2}^{\prime} \sigma_{1}=2 \sigma_{3}+2 \sigma_{3}^{\prime}, \quad \sigma_{5}^{\prime} \sigma_{1}=3 \sigma_{6}+2 \sigma_{6}^{\prime}+q \sigma_{2}, \\
& \sigma_{3} \sigma_{1}=2 \sigma_{4}+2 \sigma_{4}^{\prime}+2 q, \quad \sigma_{6} \sigma_{1}=\sigma_{7}+q \sigma_{3}^{\prime}, \\
& \sigma_{3}^{\prime} \sigma_{1}=\sigma_{4}^{\prime}+\sigma_{4}^{\prime \prime}, \quad \sigma_{6}^{\prime} \sigma_{1}=\sigma_{7}+q \sigma_{3}, \\
& \sigma_{4} \sigma_{1}=2 \sigma_{5}+q \sigma_{1}, \quad \sigma_{7} \sigma_{1}=\sigma_{8}+q \sigma_{4}+q \sigma_{4}^{\prime}, \\
& \sigma_{4}^{\prime} \sigma_{1}=2 \sigma_{5}+\sigma_{5}^{\prime}+q \sigma_{1}, \quad \sigma_{8} \sigma_{1}=2 q \sigma_{5}, \\
& \sigma_{2} \sigma_{2}=\sigma_{4}+2 \sigma_{4}^{\prime}+2 \sigma_{4}^{\prime \prime}, \\
& \sigma_{2}^{\prime} \sigma_{2}=\sigma_{4}+3 \sigma_{4}^{\prime}+\sigma_{4}^{\prime \prime}+2 q \text {, } \\
& \sigma_{3} \sigma_{2}=3 \sigma_{5}+\sigma_{5}^{\prime}+3 q \sigma_{1}, \\
& \sigma_{3}^{\prime} \sigma_{2}=\sigma_{5}+\sigma_{5}^{\prime} \\
& \sigma_{4} \sigma_{2}=\sigma_{6}+\sigma_{6}^{\prime}+2 q \sigma_{2}^{\prime}, \\
& \sigma_{4}^{\prime} \sigma_{2}=2 \sigma_{6}+3 \sigma_{6}^{\prime}+q \sigma_{2}+q \sigma_{2}^{\prime}, \\
& \sigma_{4}^{\prime \prime} \sigma_{2}=2 \sigma_{6}+\sigma_{6}^{\prime}, \\
& \sigma_{5} \sigma_{2}=\sigma_{7}+2 q \sigma_{3}+q \sigma_{3}^{\prime}, \\
& \sigma_{5}^{\prime} \sigma_{2}=3 \sigma_{7}+q \sigma_{3}+2 q \sigma_{3}^{\prime} \text {, } \\
& \sigma_{6} \sigma_{2}=\sigma_{8}+q \sigma_{4}^{\prime} \text {, } \\
& \sigma_{6}^{\prime} \sigma_{2}=2 q \sigma_{4}+q \sigma_{4}^{\prime}+q^{2} \\
& \sigma_{7} \sigma_{2}=3 q \sigma_{5}+q^{2} \sigma_{1}, \\
& \sigma_{8} \sigma_{2}=2 q \sigma_{6}^{\prime}+q^{2} \sigma_{2}^{\prime} \text {, } \\
& \sigma_{2}^{\prime} \sigma_{2}^{\prime}=3 \sigma_{4}+3 \sigma_{4}^{\prime}+\sigma_{4}^{\prime \prime}+2 q, \\
& \sigma_{2}^{\prime} \sigma_{3}=5 \sigma_{5}+\sigma_{5}^{\prime}+3 q \sigma_{1}, \\
& \sigma_{2}^{\prime} \sigma_{3}^{\prime}=\sigma_{5}+\sigma_{5}^{\prime}+q \sigma_{1}, \\
& \sigma_{2}^{\prime} \sigma_{4}=2 \sigma_{6}+3 \sigma_{6}^{\prime}+q \sigma_{2}+q \sigma_{2}^{\prime}, \\
& \sigma_{2}^{\prime} \sigma_{4}^{\prime}=3 \sigma_{6}+3 \sigma_{6}^{\prime}+q \sigma_{2}+2 q \sigma_{2}^{\prime} \text {, } \\
& \sigma_{2}^{\prime} \sigma_{4}^{\prime \prime}=\sigma_{6}+\sigma_{6}^{\prime}+q \sigma_{2}, \\
& \sigma_{2}^{\prime} \sigma_{5}=2 \sigma_{7}+2 q \sigma_{3}+2 q \sigma_{3}^{\prime} \text {, } \\
& \sigma_{2}^{\prime} \sigma_{5}^{\prime}=2 \sigma_{7}+2 q \sigma_{3}+4 q \sigma_{3}^{\prime} \text {, } \\
& \sigma_{2}^{\prime} \sigma_{6}=q \sigma_{4}+q \sigma_{4}^{\prime}+q \sigma_{4}^{\prime \prime}, \\
& \sigma_{2}^{\prime} \sigma_{6}^{\prime}=\sigma_{8}+q \sigma_{4}+2 q \sigma_{4}^{\prime}+q^{2}, \\
& \sigma_{2}^{\prime} \sigma_{7}=3 q \sigma_{5}+q \sigma_{5}^{\prime}+q^{2} \sigma_{1}, \\
& \sigma_{2}^{\prime} \sigma_{8}=2 q \sigma_{6}+2 q \sigma_{6}^{\prime}+q^{2} \sigma_{2}^{\prime} \text {, } \\
& \sigma_{3} \sigma_{3}=3 \sigma_{6}+5 \sigma_{6}^{\prime}+q \sigma_{2}+3 q \sigma_{2}^{\prime} \text {, } \\
& \sigma_{3}^{\prime} \sigma_{3}=\sigma_{6}+\sigma_{6}^{\prime}+q \sigma_{2}+q \sigma_{2}^{\prime}, \\
& \sigma_{3}^{\prime} \sigma_{3}^{\prime}=\sigma_{6}+\sigma_{6}^{\prime}, \\
& \sigma_{4} \sigma_{3}=2 \sigma_{7}+2 q \sigma_{3}+2 q \sigma_{3}^{\prime} \text {, } \\
& \sigma_{3}^{\prime} \sigma_{4}=q \sigma_{3}+q \sigma_{3}^{\prime} \\
& \sigma_{4}^{\prime} \sigma_{3}=2 \sigma_{7}+3 q \sigma_{3}+4 q \sigma_{3}^{\prime} \text {, } \\
& \sigma_{4}^{\prime \prime} \sigma_{3}=q \sigma_{3}+2 q \sigma_{3}^{\prime}, \\
& \sigma_{3}^{\prime} \sigma_{4}^{\prime}=\sigma_{7}+q \sigma_{3}+q \sigma_{3}^{\prime} \text {, } \\
& \sigma_{3}^{\prime} \sigma_{4}^{\prime \prime}=\sigma_{7} \\
& \sigma_{5} \sigma_{3}=\sigma_{8}+2 q \sigma_{4}+3 q \sigma_{4}^{\prime}+q \sigma_{4}^{\prime \prime}+q^{2} \text {, } \\
& \sigma_{3}^{\prime} \sigma_{5}=q \sigma_{4}+q \sigma_{4}^{\prime}+q^{2} \\
& \sigma_{5}^{\prime} \sigma_{3}=2 q \sigma_{4}+4 q \sigma_{4}^{\prime}+2 q \sigma_{4}^{\prime \prime}+2 q^{2}, \\
& \sigma_{3}^{\prime} \sigma_{5}^{\prime}=\sigma_{8}+q \sigma_{4}+q \sigma_{4}^{\prime} \text {, } \\
& \sigma_{6} \sigma_{3}=q \sigma_{5}+q \sigma_{5}^{\prime}+q^{2} \sigma_{1}, \\
& \sigma_{3}^{\prime} \sigma_{6}=q \sigma_{5}, \\
& \sigma_{6}^{\prime} \sigma_{3}=3 q \sigma_{5}+q \sigma_{5}^{\prime}+q^{2} \sigma_{1}, \\
& \sigma_{3}^{\prime} \sigma_{6}^{\prime}=q \sigma_{5}+q^{2} \sigma_{1} \\
& \sigma_{7} \sigma_{3}=3 q \sigma_{6}+3 q \sigma_{6}^{\prime}+q^{2} \sigma_{2}+q^{2} \sigma_{2}^{\prime} \text {, } \\
& \sigma_{3}^{\prime} \sigma_{7}=q \sigma_{6}^{\prime}+q^{2} \sigma_{2}^{\prime} \text {, } \\
& \sigma_{8} \sigma_{3}=2 q \sigma_{7}+q^{2} \sigma_{3}+2 q^{2} \sigma_{3}^{\prime}, \\
& \sigma_{3}^{\prime} \sigma_{8}=q^{2} \sigma_{3}
\end{aligned}
$$




$$
\begin{aligned}
& \sigma_{4} \sigma_{4}=\sigma_{8}+2 q \sigma_{4}^{\prime}+q^{2}, \quad \sigma_{4} \sigma_{4}^{\prime}=2 q \sigma_{4}+2 q \sigma_{4}^{\prime}+q \sigma_{4}^{\prime \prime}+q^{2}, \\
& \sigma_{4}^{\prime} \sigma_{4}^{\prime}=\sigma_{8}+2 q \sigma_{4}+3 q \sigma_{4}^{\prime}+q \sigma_{4}^{\prime \prime}+2 q^{2}, \quad \sigma_{4} \sigma_{4}^{\prime \prime}=q \sigma_{4}^{\prime}+q^{2} \text {, } \\
& \sigma_{4}^{\prime \prime} \sigma_{4}^{\prime \prime}=\sigma_{8}, \quad \sigma_{4}^{\prime} \sigma_{4}^{\prime \prime}=q \sigma_{4}+q \sigma_{4}^{\prime} . \\
& \sigma_{4} \sigma_{5}=2 q \sigma_{5}+q \sigma_{5}^{\prime}+q^{2} \sigma_{1}, \quad \sigma_{5}^{\prime} \sigma_{4}=2 q \sigma_{5}+q \sigma_{5}^{\prime}+2 q^{2} \sigma_{1}, \\
& \sigma_{4}^{\prime} \sigma_{5}=3 q \sigma_{5}+q \sigma_{5}^{\prime}+2 q^{2} \sigma_{1}, \quad \sigma_{5}^{\prime} \sigma_{4}^{\prime}=4 q \sigma_{5}+q \sigma_{5}^{\prime}+2 q^{2} \sigma_{1}, \\
& \sigma_{4}^{\prime \prime} \sigma_{5}=q \sigma_{5}+q^{2} \sigma_{1}, \quad \sigma_{5}^{\prime} \sigma_{4}^{\prime \prime}=2 q \sigma_{5}, \\
& \sigma_{4} \sigma_{6}=q \sigma_{6}^{\prime}+q^{2} \sigma_{2}, \quad \sigma_{6}^{\prime} \sigma_{4}=2 q \sigma_{6}+q \sigma_{6}^{\prime}+q^{2} \sigma_{2}^{\prime}, \\
& \sigma_{4}^{\prime} \sigma_{6}=q \sigma_{6}+q \sigma_{6}^{\prime}+q^{2} \sigma_{2}^{\prime}, \quad \sigma_{6}^{\prime} \sigma_{4}^{\prime}=q \sigma_{6}+2 q \sigma_{6}^{\prime}+q^{2} \sigma_{2}+q^{2} \sigma_{2}^{\prime}, \\
& \sigma_{4}^{\prime \prime} \sigma_{6}=q \sigma_{6}^{\prime}, \quad \sigma_{6}^{\prime} \sigma_{4}^{\prime \prime}=q^{2} \sigma_{2}^{\prime}, \\
& \sigma_{4} \sigma_{7}=q \sigma_{7}+q^{2} \sigma_{3}+2 q^{2} \sigma_{3}^{\prime}, \quad \sigma_{8} \sigma_{4}=q^{2} \sigma_{4}+q^{2} \sigma_{4}^{\prime}+q^{2} \sigma_{4}^{\prime \prime}, \\
& \sigma_{4}^{\prime} \sigma_{7}=q \sigma_{7}+2 q^{2} \sigma_{3}+2 q^{2} \sigma_{3}^{\prime}, \quad \sigma_{8} \sigma_{4}^{\prime}=q^{2} \sigma_{4}+2 q^{2} \sigma_{4}^{\prime}+q^{3}, \\
& \sigma_{4}^{\prime \prime} \sigma_{7}=q^{2} \sigma_{3}, \quad \sigma_{8} \sigma_{4}^{\prime \prime}=q^{2} \sigma_{4}+q^{3}, \\
& \sigma_{5} \sigma_{5}=2 q \sigma_{6}+2 q \sigma_{6}^{\prime}+q^{2} \sigma_{2}+q^{2} \sigma_{2}^{\prime}, \quad \sigma_{6} \sigma_{6}=q^{2} \sigma_{4} \text {, } \\
& \sigma_{5}^{\prime} \sigma_{5}=q \sigma_{6}+2 q \sigma_{6}^{\prime}+q^{2} \sigma_{2}+2 q^{2} \sigma_{2}^{\prime}, \quad \sigma_{6}^{\prime} \sigma_{6}=q^{2} \sigma_{4}^{\prime}+q^{3} \text {, } \\
& \sigma_{5}^{\prime} \sigma_{5}^{\prime}=2 q \sigma_{6}+4 q \sigma_{6}^{\prime}+2 q^{2} \sigma_{2}^{\prime}, \quad \sigma_{6}^{\prime} \sigma_{6}^{\prime}=q^{2} \sigma_{4}+q^{2} \sigma_{4}^{\prime}+q^{2} \sigma_{4}^{\prime \prime}, \\
& \sigma_{6} \sigma_{5}=q^{2} \sigma_{3}+q^{2} \sigma_{3}^{\prime}, \quad \sigma_{5}^{\prime} \sigma_{6}=q \sigma_{7}+q^{2} \sigma_{3}, \\
& \sigma_{6}^{\prime} \sigma_{5}=q \sigma_{7}+q^{2} \sigma_{3}+2 q^{2} \sigma_{3}^{\prime}, \quad \sigma_{5}^{\prime} \sigma_{6}^{\prime}=2 q^{2} \sigma_{3}+2 q^{2} \sigma_{3}^{\prime}, \\
& \sigma_{7} \sigma_{5}=q^{2} \sigma_{4}+2 q^{2} \sigma_{4}^{\prime}+q^{2} \sigma_{4}^{\prime \prime}+q^{3}, \quad \sigma_{5}^{\prime} \sigma_{7}=2 q^{2} \sigma_{4}+2 q^{2} \sigma_{4}^{\prime}+2 q^{3} \text {, } \\
& \sigma_{8} \sigma_{5}=q^{2} \sigma_{5}+q^{2} \sigma_{5}^{\prime}+q^{3} \sigma_{1}, \quad \sigma_{5}^{\prime} \sigma_{8}=2 q^{2} \sigma_{5}+2 q^{3} \sigma_{1}, \\
& \sigma_{7} \sigma_{6}=q^{2} \sigma_{5}+q^{3} \sigma_{1}, \quad \sigma_{6}^{\prime} \sigma_{7}=q^{2} \sigma_{5}+q^{2} \sigma_{5}^{\prime}+q^{3} \sigma_{1}, \\
& \sigma_{8} \sigma_{6}=q^{3} \sigma_{2}^{\prime}, \quad \sigma_{6}^{\prime} \sigma_{8}=q^{2} \sigma_{6}+q^{2} \sigma_{6}^{\prime}+q^{3} \sigma_{2}, \\
& \sigma_{7} \sigma_{7}=q^{2} \sigma_{6}+q^{2} \sigma_{6}^{\prime}+q^{3} \sigma_{2}+q^{3} \sigma_{2}^{\prime}, \quad \sigma_{7} \sigma_{8}=q^{3} \sigma_{3}+2 q^{3} \sigma_{3}^{\prime}, \\
& \sigma_{8} \sigma_{8}=q^{3} \sigma_{4}^{\prime}+q^{3} \sigma_{4}^{\prime \prime}+q^{4} \text {. }
\end{aligned}
$$

Vladimiro Benedetti

Département de mathématiques et applications, ENS

CNRS, PSL University, 75005 Paris, France.

Vladimiro.Benedetti@ens.fr

Laurent MANIVEL

Institut de Mathématiques de Toulouse, UMR 5219

CNRS, UPS, F-31062 Toulouse Cedex 9, France.

manivel@math.cnrs.fr 


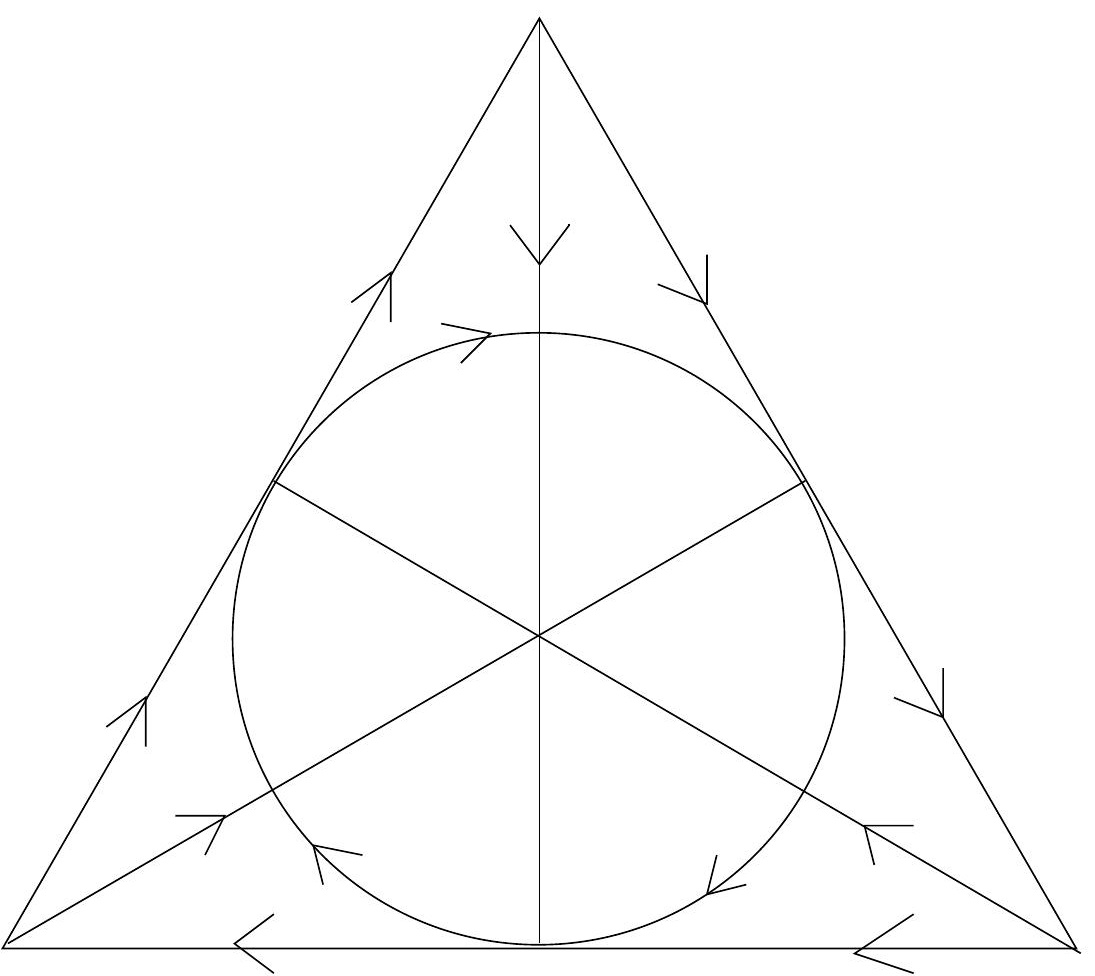

